\title{
Spatial Modulation Concept for Massive Multiuser MIMO Systems
}

\author{
Khaled M. Humadi, Ahmed Iyanda Sulyman, and Abdulhameed Alsanie \\ Department of Electrical Engineering, King Saud University, Riyadh 11421, Saudi Arabia \\ Correspondence should be addressed to Ahmed Iyanda Sulyman; asulyman@ksu.edu.sa
}

Received 18 February 2014; Accepted 18 May 2014; Published 9 June 2014

Academic Editor: Shahram Yousefi

Copyright ( 2014 Khaled M. Humadi et al. This is an open access article distributed under the Creative Commons Attribution License, which permits unrestricted use, distribution, and reproduction in any medium, provided the original work is properly cited.

This paper presents the concept of spatial modulation (SM) scheme for massive multiuser MIMO (MU-MIMO) system. We consider a MU-MIMO system where $K$ users, each equipped with multiple antennas, are jointly serviced by a multiantenna base station transmitter (BSTx) using appropriate precoding scheme at the BSTx. The main idea introduced here is the utilization of the user's subchannel index corresponding to the precoding matrix used at the BSTx, to convey extra useful information. This idea has not been explored, and it provides significant throughput enhancements in a multiuser system with large number of users. We examine the performance of the proposed scheme by numerical simulations. The results show that as the number of users and the receiving antennas for each user increase, the overall system throughput gets better, albeit at the cost of some degradation in the BER performance due to interantenna interference (IAI) experienced at the receiver. We then explore zero-padding approach that helps to remove these IAI, in order to alleviate the BER degradations.

\section{Introduction}

Massive multiuser multiple input multiple output (MIMO) systems have gained significant research attentions lately because they provide significant boost in the capacity of MIMO systems [1-3]. Multiuser MIMO systems have been investigated for long time now. However, a recent new development in this research area is the aggressive use of very large number of antennas, known as massive multiuser MIMO systems. Currently in the fourth-generation (4G) long-term evolution (LTE) for cellular system, the use of up to $8 \times 8$ MIMO systems have been standardized, both for single-user and multiuser systems. It is hoped however that massive MIMO systems with hundreds of antennas at the base station (BS) will eventually be standardized in the fifth-generation $(5 \mathrm{G})$ cellular system, as part of the major data rate enhancement techniques to be introduced in $5 \mathrm{G}$ [4]. To this end, several research efforts have been devoted to studying the benefits of massive MIMO systems under different considerations.

Spatial modulation (SM) is another new promising transmission technique that uses antenna indexes in a multiple antenna system, as additional means of data transmissions. The main idea behind spatial modulation is to use the index of the active antennas at any time instant, transmitting or receiving antenna depending on whether the spatial modulation scheme is applied at the transmitter or at the receiver, to convey extra information. Thus, the information bits to be transmitted are divided into blocks of two parts [5]. The first part is mapped to a symbol chosen from the signaling constellation, where the number of bits per symbol depends on the type of modulation used. The second part determines the index of the antenna to be selected from a set of antennas available for data transmission or reception. Therefore, unlike antenna selection in the conventional MIMO systems which depends on the channel states and the received signal strength, antenna selection in spatial modulation depends on the incoming user data stream $[6,7]$.

Spatial modulation schemes were first introduced in $[8$, 9], where the principle of wireless transmission in which the information is carried by both the index of the active antenna and the symbol transmitted through this active antenna was illustrated. In [10], then, the idea of space shift keying (SSK) modulation was introduced as a modulation 
scheme, which uses only the spatial modulation concept. In the SSK scheme, there were no transmitted symbols. Only the antennas' indices were used to convey information. Because no symbols were transmitted, SSK reduces the system complexity by removing the amplitude/phase modulation (APM) required in the transmission and detection components, but at the expense of some degradation in the system's spectral efficiency. Since only one antenna is active at a time in the SSK scheme, the scheme exhibits no interantenna interference (IAI), just like a single antenna wireless system. In [11], a combination of spatial modulation (SM) and space-time block coding (STBC) were considered, in order to take advantage of the benefits of both schemes. A generalized version of SM called generalized spatial modulation (GSM) system with multiple active transmitting antennas (MA-SM) and low complexity detection scheme was introduced in [12]. In the GSM system, more than one transmitting antennas are active at the same time which increases the system spectral efficiency. In [13], Rong Zhang proposed a spatial modulation (SM) scheme at the receiver side for single user MIMO system called generalised precoding aided spatial modulation (GPASM).

In this paper, we propose the concept of spatial modulation (SM) at the receiver side for multiuser MIMO (MUMIMO) system. Our work can be considered a generalization of the work in [13] to the case of multiuser system. We study the performance of the proposed scheme by simulation, and we demonstrate that significant throughput enhancement can be obtained using the proposed scheme.

\section{System Model and Analysis}

Consider a downlink multiuser MIMO system shown in Figure 1 in which a base station transmitter (BSTx) with $N_{t}$ transmitting antennas communicates simultaneously with $K$ independent users on the same time-frequency resources. Each user is equipped with $N_{r}$ receiving antennas and assuming that $N_{t}>N_{r}$. At any transmission time instance, the data for each user is divided into blocks of $n+p$ bits where $n=\log _{2} M$ ( $M$ is the symbol constellation size) and $p=\log _{2} N_{r}$. The first $n$ bits $\left[\begin{array}{llll}b_{1} & b_{2} & \cdots & b_{n}\end{array}\right]$ are mapped to a corresponding symbol in the constellation, while the next $p$ bits $\left[\begin{array}{llll}b_{n+1} & b_{n+2} & \cdots & b_{n+p}\end{array}\right]$ are used to activate a particular receiving antennas. For simplicity of representation, we have considered here only the case where one receiving antenna is switched for each user. However our works and results are easily extended to cases where two or more antennas are activated per user. At the receiver side, when the user receives the correct symbol, the first $n$ bits of the user data transmitted by the BSTx over a particular receiving antenna will be decoded using maximum likelihood (ML) estimate of the received signal, while the next $p$ bits are added based on the index of the antenna from where the signal (or symbol) is received or detected. Thus, the index of this antenna also conveys useful information in addition to the transmitted symbol.

Let the vector $\mathbf{x}=\left[x_{m, j}^{1}, x_{m, j}^{2}, \ldots, x_{m, j}^{K}\right]^{T}$ represent the transmitted super symbols from the BSTx to all users, where the notation $x_{m, j}^{k}$ indicates that the BSTx transmits a modulated symbol $s_{m} \in\left\{s_{1}, s_{2} \ldots, s_{M}\right\}$ to the $k$ th user, and the symbol is to be received at the $j$ th receiving antenna of the user, $j=1,2, \ldots, N_{r}$. We first assume that the channel is totally uncorrelated and that the channel state information (CSI) is available at the transmitter side. The MU-MIMO channel matrix $\mathbf{H}$ for this system can be written as

$$
\mathbf{H}=\left[\begin{array}{c}
\mathbf{H}^{1} \\
\mathbf{H}^{2} \\
\vdots \\
\mathbf{H}^{K}
\end{array}\right]
$$

where $\mathbf{H}^{k}, k=1,2, \ldots, K$ is the $N_{r} \times N_{t}$ channel matrix corresponding to the $k$ th user and is given by

$$
\mathbf{H}^{k}=\left[\begin{array}{cccc}
h_{11}^{k} & h_{12}^{k} & \ldots & h_{1 N_{t}}^{k} \\
h_{21}^{k} & h_{22}^{k} & \ldots & h_{2 N_{t}}^{k} \\
& & \vdots & \\
& & & \\
h_{N_{r} 1}^{k} & h_{N_{r} 2}^{k} & \ldots & h_{N_{r} N_{t}}^{k}
\end{array}\right] .
$$

Now we will discuss two methods proposed here to activate the receiving antenna for each user, named here the "subchannel selection" method and the "zero-padding" method.

2.1. Subchannel Selection Method. The main idea of this method is the utilization of users' subchannels as a means of additional data transmission, by collecting the rows of user channel matrices into a multiuser precoding matrix used at the BSTx. For the case when one antenna is switched per user, one row is taken from a user channel matrix at a time. If more than one antenna are needed to be switched, then the corresponding number of rows are taken. After the transmitter precoding operations, the resulting transmitted vector $\widehat{\mathbf{x}} \in \mathbb{C}^{N_{t} \times 1}$ can be written as

$$
\widehat{\mathbf{x}}=\mathbf{G} \mathbf{x},
$$

where $\mathbf{G} \in \mathbb{C}^{N_{t} \times K}$ is the multiuser precoding matrix for the current transmitted symbols which is determined by the $p$ bits subblocks for all users. The received vector $\mathbf{y} \in \mathbb{C}^{N_{r} K \times 1}$ may be written as

$$
\mathbf{y}=\mathbf{H} \widehat{\mathbf{x}}+\mathbf{w},
$$

where $\mathbf{w} \in \mathbb{C}^{N_{r} K \times 1}$ is the Gaussian noise vector with all its elements having a zero mean and a variance of $\sigma^{2}=$ $N_{0} / 2$. The precoding is applied in a way that it can eliminate the effects of channel fading and multiuser interference at the desired receiving antennas. This can be achieved using either minimum mean square error (MMSE) precoding or zero forcing (ZF) precoding. In this paper, we choose ZF precoding in which the precoding matrix is given by

$$
\mathbf{G}=\beta \mathbf{H}_{s}^{H}\left(\mathbf{H}_{s} \mathbf{H}_{s}^{H}\right)^{-1},
$$

where $(\cdot)^{H}$ represents the conjugate transpose of a matrix and $\mathbf{H}_{s} \in \mathbb{C}^{K \times N_{t}}$ is the subchannel selected from $\mathbf{H}$ as enumerated above (and also explained in detail later), and $\beta$ is 


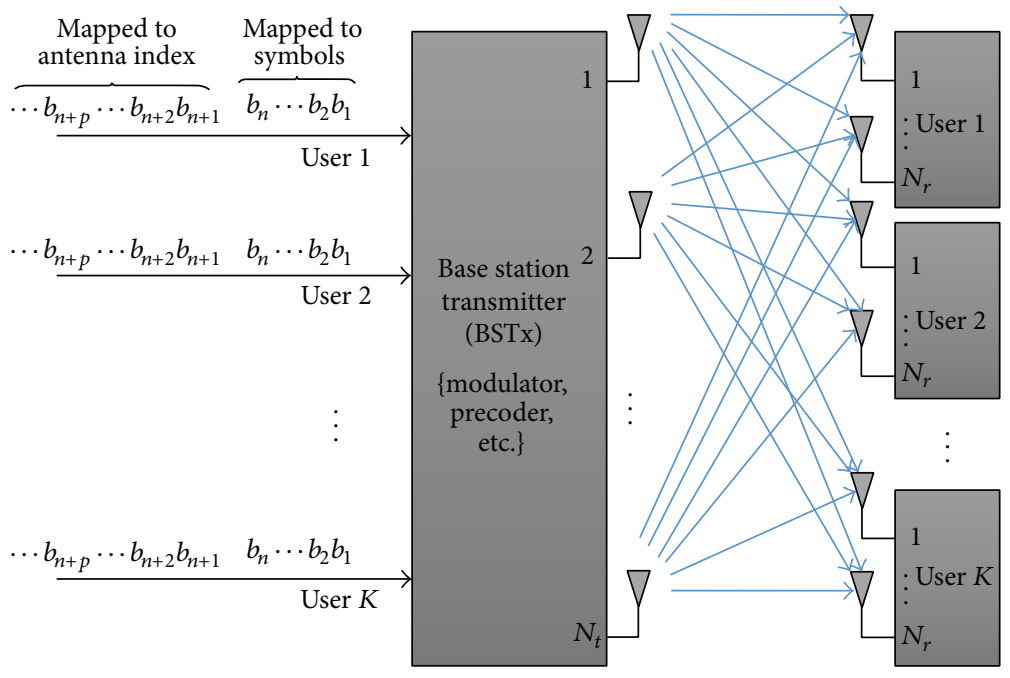

FIGURE 1: SM for multiuser MIMO System.

a normalization factor introduced in order to meet the total transmitted power constraint after precoding. This factor is given by

$$
\beta=\sqrt{\frac{N_{t}}{\operatorname{tr}\left[\left(\mathbf{H}_{s} \mathbf{H}_{s}^{H}\right)^{-1}\right]}},
$$

where $\operatorname{tr}[\cdot]$ denotes the trace of a matrix. If the transmitted symbol of the $k$ th user is precoded at the BSTx such that the fading-free data is received at the $j$ th antenna of the user, then the vector $\mathbf{y}^{k} \in \mathbb{C}^{N_{r} \times 1}$ received by the $k$ th user can be described as

$$
\begin{gathered}
y^{k}=\beta x_{m, \hat{j}}^{k}+w_{\hat{j}}^{k}, \quad \hat{j}=j, \\
y^{k}=v_{\hat{j}}^{k}+w_{\hat{j}}^{k}, \quad \hat{j} \neq j,
\end{gathered}
$$

where $v_{\hat{j}}^{k}=\sum_{i=1}^{N_{t}} h_{\hat{j} i}^{k} \widehat{x}_{i}, \widehat{j} \neq j$ is the effect of channel fading and multiuser interference, while $w_{\hat{j}}^{k}$ represents the Gaussian noise for the $k$ th user at the $\hat{j}$ th receiving antenna. For each user, the ML detector computes the Euclidean distances between the received signal and the set of possible super symbols $\mathbf{x}_{m, j}^{k} \in\left\{x_{m, 1}^{k}, x_{m, 2}^{k}, \ldots, x_{m, N_{r}}^{k}\right\} ; m=1,2, \ldots, M$ transmitted. Then, the ML detection operation at each user receiving device can be expressed as

$$
[\widehat{j}, \widehat{m}]=\arg \min _{\substack{m \in\{1,2, . ., M\} \\ j \in\left\{1,2, . ., N_{r}\right\}}}\left\{\left\|\frac{1}{\beta} \mathbf{y}^{k}-\mathbf{x}_{m, j}^{k}\right\|^{2}\right\},
$$

where $\widehat{m}$ is the argument of the symbol $s_{\widehat{m}}$ in the constellation that gives the minimum distance, while $\hat{j}$ is the antenna index at which the ML detector gets the minimum distance. The correct decision is obtained when $\widehat{m}=m$ and $\hat{j}=j$.

Selecting the Subchannel. Subchannel $\mathbf{H}_{s}$ is selected in a way that the precoding operation at the BSTx can remove the effect of channel fading and multiuser interference at the desired receiving antennas of the users. For simplicity of illustration, we considered the case where only one antenna is switched per user here. In this case, we need to receive the correct data at only one receiving antenna per user. This can be obtained by choosing the elements of the subchannel $\mathbf{H}_{s}$ from the MU-MIMO channel $\mathbf{H}$ such that the $k$ th row of $\mathbf{H}_{s}$ is selected from the $k$ th user channel $\mathbf{H}^{k}$ according to the $p$ subblock in the user data. Therefore, the total number of available subchannels is $2^{K p}=\left(N_{r}\right)^{K}$.

Example 1. As an illustration of the subchannel selection method,consider a system with two users $(K=2)$, four transmitting antennas $\left(N_{t}=4\right)$, and two receiving antennas for each user $\left(N_{r}=2\right)$ as shown in Figure 2. The MU-MIMO channel matrix $\mathbf{H}$ can be written as

$$
\mathbf{H}=\left[\begin{array}{l}
\mathbf{H}^{1} \\
\mathbf{H}^{2}
\end{array}\right]=\left[\begin{array}{llll}
h_{11}^{1} & h_{12}^{1} & h_{13}^{1} & h_{14}^{1} \\
h_{21}^{1} & h_{22}^{1} & h_{23}^{1} & h_{24}^{1} \\
h_{11}^{2} & h_{12}^{2} & h_{13}^{2} & h_{14}^{2} \\
h_{21}^{2} & h_{22}^{2} & h_{23}^{2} & h_{24}^{2}
\end{array}\right]
$$

where $\mathbf{H}^{1}$ and $\mathbf{H}^{2}$ are the channel matrices for user 1 and user 2, respectively. If we assume that QPSK modulation format is used at the transmitter, the incoming data for each user is divided into blocks of 3 bits. The first 2 bits are mapped onto the appropriate QPSK symbol, while the third bit for each user determines which row from the user channel matrix $\mathbf{H}^{k}$ is selected to constitute the subchannel $\mathbf{H}_{s}$ to be used as percoding matrix for the transmitted symbols. Instead of determining the subchannel matrix $\mathbf{H}_{s}$ according to the $p$ subblock for each user separately, we group the subblock $p$ for all users into subchannel selection code $C \in\{00,01,10,11\}$ and then select $\mathbf{H}_{s}$ according to the code $C$ at any time instant. 


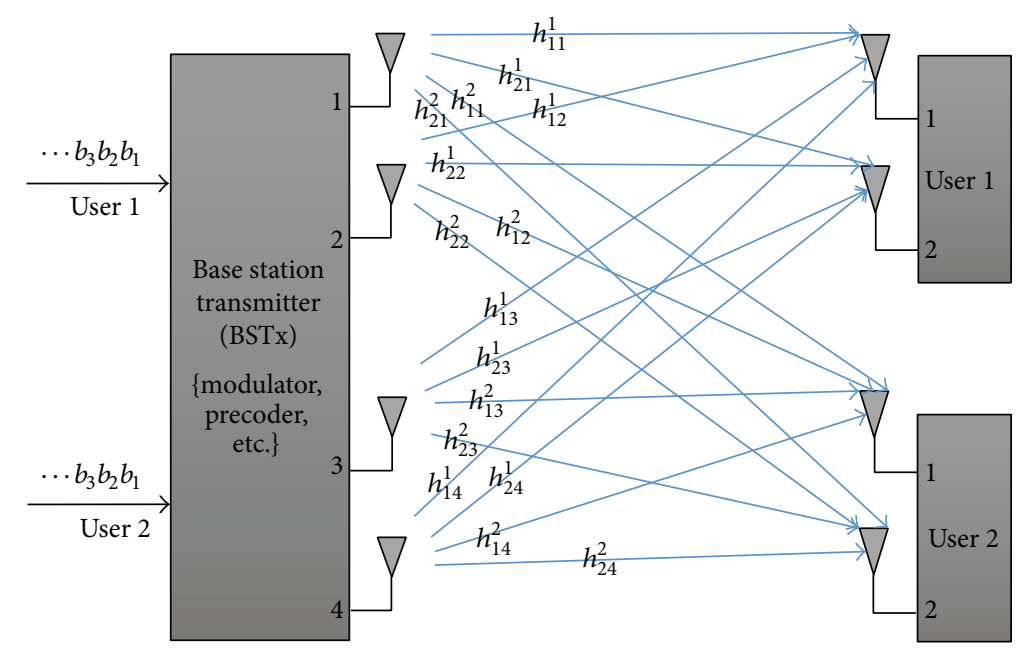

FIgURE 2: Example of SM for MU-MIMO system with $K=2, N_{t}=4$, and $N_{r}=2$.

The number of the available subchannels is given by $\left(N_{r}\right)^{K} \equiv$ 4 for this example with $\mathbf{H}_{s} ; s=1, \ldots, 4$ is given by

$$
\begin{array}{ll}
\mathbf{H}_{1}=\left[\begin{array}{llll}
h_{11}^{1} & h_{12}^{1} & h_{13}^{1} & h_{14}^{1} \\
h_{11}^{2} & h_{12}^{2} & h_{13}^{2} & h_{14}^{2}
\end{array}\right], & \mathbf{H}_{2}=\left[\begin{array}{llll}
h_{11}^{1} & h_{12}^{1} & h_{13}^{1} & h_{14}^{1} \\
h_{21}^{2} & h_{22}^{2} & h_{23}^{2} & h_{24}^{2}
\end{array}\right] \\
\mathbf{H}_{3}=\left[\begin{array}{llll}
h_{21}^{1} & h_{22}^{1} & h_{23}^{1} & h_{24}^{1} \\
h_{11}^{2} & h_{12}^{2} & h_{13}^{2} & h_{14}^{2}
\end{array}\right], & \mathbf{H}_{4}=\left[\begin{array}{llll}
h_{21}^{1} & h_{22}^{1} & h_{23}^{1} & h_{24}^{1} \\
h_{21}^{2} & h_{22}^{2} & h_{23}^{2} & h_{24}^{2}
\end{array}\right] .
\end{array}
$$

The code $C$ is mapped to the subchannel sets as follows:

$$
\begin{aligned}
& C=00 \longrightarrow \mathbf{H}_{1}, \quad C=01 \longrightarrow \mathbf{H}_{2} \\
& C=10 \longrightarrow \mathbf{H}_{3}, \quad C=11 \longrightarrow \mathbf{H}_{4} .
\end{aligned}
$$

At the receiver side, after the ML detection for each user, if the symbol is detected at the first receiving antenna, then $b_{3}=0$, and if the symbol is detected at the second antenna, $b_{3}=1$.

2.2. Zero-Padding Method. In the subchannel selection method, we activated the desired receiving antennas for all users by choosing the appropriate elements of the subchannel matrix $\mathbf{H}_{s}$ to precode the input data at the BSTx in order to eliminate the effect of channel fading and multiuser interference at the desired receiving antennas for the users. However the other antennas are affected by these channel impairments. Now we will activate the receiving antennas by zero-padding method such that all receiving antennas for each user will receive zeros, except for the activated antennas which will receive the transmitted symbols. In this way, we can totally cancel the effect of channel fading and multiuser interference on the received data by precoding the zeropadded input data using the MU-MIMO channel matrix $\mathbf{H}$ (assuming $\mathbf{H}$ is available at the transmitter side). Consider the general system in Figure 1, after zero-padding the input vector $\mathbf{x}$, we will get a vector $\mathbf{x}_{\mathrm{zp}}=\left[\mathbf{x}_{\mathrm{zp}}^{1}, \mathbf{x}_{\mathrm{zp}}^{2}, \ldots, \mathbf{x}_{\mathrm{zp}}^{K}\right]^{T}$ where $\mathbf{x}_{\mathrm{zp}}^{k} \in \mathbb{C}^{1 \times N_{r}}$ denotes the zero-padded vector corresponding to the $k$ th user. For the case where only one receiving antenna is activated per user, this can be written as

$$
\mathbf{x}_{\mathrm{zp}}^{k}=[\underbrace{0,0, \ldots, 0}_{j-1}, x_{m, j}^{k}, \underbrace{0,0, \ldots, 0}_{N_{r}-j}] .
$$

After zero-forcing precoding, the transmitted vector $\widehat{\mathbf{x}}_{\mathrm{zp}} \in$ $\mathbb{C}^{N_{t} \times 1}$ can be written as

$$
\widehat{\mathbf{x}}_{\mathrm{zp}}=\mathbf{G} \mathbf{x}_{\mathrm{zp}}
$$

where, in this case, $\mathbf{G}=\beta \mathbf{H}^{H}\left(\mathbf{H} \mathbf{H}^{H}\right)^{-1}$ and $\beta=$ $\sqrt{N_{t} / \operatorname{tr}\left[\left(\mathbf{H H}^{H}\right)^{-1}\right]}$. The received vector $\mathbf{y} \in \mathbb{C}^{N_{r} K \times 1}$ may be written as

$$
\mathbf{y}=\mathbf{H} \widehat{\mathbf{x}}_{\mathrm{zp}}+\mathbf{w}
$$

where $\mathbf{w}$ is defined in (4). The received vector $\mathbf{y}^{k} \in \mathbb{C}^{N_{r} \times 1}$ for the $k$ th user can be described as

$$
\begin{gathered}
y^{k}=\beta x_{m, \hat{j}}^{k}+w_{\hat{j}}^{k}, \quad \hat{j}=j, \\
y^{k}=0+w_{\hat{j}}^{k}, \quad \widehat{j} \neq j,
\end{gathered}
$$

where $w_{\hat{j}}^{k}$ is defined in (7). From (15), it is obvious that for each user, only one receiving antenna will receive the transmitted symbol and the other antennas will receive zeros. The ML detector is then applied as in (8).

Example 2. As an illustration of the zero-padding method, consider the system in Figure 2 with the same information 
as in Example 1. Here the code $C$ determines how the input vector $\mathbf{x}$ is zero-padded to obtain $\mathbf{x}_{\mathrm{zp}}$ as

$$
\begin{aligned}
& C=00 \longrightarrow \mathbf{x}_{\mathrm{zp}}=\left[x_{m, 1}^{1}, 0, x_{m, 1}^{2}, 0\right], \\
& C=01 \longrightarrow \mathbf{x}_{\mathrm{zp}}=\left[x_{m, 1}^{1}, 0,0, x_{m, 2}^{2}\right], \\
& C=10 \longrightarrow \mathbf{x}_{\mathrm{zp}}=\left[0, x_{m, 2}^{1}, x_{m, 1}^{2}, 0\right], \\
& C=11 \longrightarrow \mathbf{x}_{\mathrm{zp}}=\left[0, x_{m, 2}^{1}, 0, x_{m, 2}^{2}\right] .
\end{aligned}
$$

The vector $\mathbf{x}_{\mathrm{zp}}$ is then precoded using the system matrix $\mathbf{H}$. For a system with $K$ users and $N_{r}$ receive antennas for each user, there will be $2^{K p}=N_{r}^{K}$ available combinations of zeropadded input vector $\mathbf{x}_{\mathrm{zp}}$.

\section{Performance Analysis}

Average Bit Error Probability Analysis. When a symbol $x_{m, j}^{k}$ is transmitted, there are three cases in which the error may occur. These are the error in the modulated symbol $s_{m}$ only, the error in the spatial symbol $j$ only, and the joint errors when both of them occur simultaneously. Therefore, as shown in [14], the average bit error probability (ABEP) can be upperbounded by the expression:

$$
\mathrm{ABEP} \leq \mathrm{ABEP}_{\text {symbol }}+\mathrm{ABEP}_{\text {spatial }}+\mathrm{ABEP}_{\text {joint }} .
$$

In [14], analytical formulas for the above three cases were presented for the case where the spatial modulation scheme is applied at the transmitter side. Each expression for the ABER was derived by summing the average pairwise error probabilities (APWEP), weighted by the corresponding average Hamming distances of the given bit mapping. The summation is taken over all the possible transmitted modulated and spatial symbols. Applying this principle at the receiver side for our case here, we get the following expressions:

$$
\begin{aligned}
& \mathrm{ABEP}_{\text {symbol }} \\
& =\frac{1}{M N_{r} \log _{2}\left(M N_{r}\right)} \\
& \times \sum_{j=1}^{N_{r}} \sum_{m=1}^{M} \sum_{\substack{\widehat{m}=1 \\
\bar{m} \neq m}}^{M} d_{H}\left(x_{m, j}^{k} \longrightarrow x_{\widehat{m}, j}^{k}\right) \operatorname{APWEP}_{\text {symbol }} \text {, } \\
& \mathrm{ABEP}_{\text {spatial }}=\frac{1}{M N_{r} \log _{2}\left(M N_{r}\right)} \\
& \times \sum_{m=1}^{M} \sum_{j=1}^{N_{r}} \sum_{\substack{\hat{j}=1 \\
\hat{j} \neq j}}^{N_{r}} d_{H}\left(x_{m, j}^{k} \longrightarrow x_{m, \hat{j}}^{k}\right) \operatorname{APWEP}_{\text {spatial }} \text {, }
\end{aligned}
$$

$$
\begin{aligned}
& \mathrm{ABEP}_{\text {joint }} \\
& =\frac{1}{M N_{r} \log _{2}\left(M N_{r}\right)} \\
& \quad \times \sum_{m=1}^{M} \sum_{j=1}^{N_{r}} \sum_{\substack{\hat{m}=1 \\
\bar{m} \neq m}}^{M} \sum_{\substack{\hat{j}=1 \\
j \neq j}}^{N_{r}} d_{H}\left(x_{m, j}^{k} \longrightarrow x_{\widehat{m}, \hat{j}}^{k}\right) \mathrm{APWEP}_{\text {joint }},
\end{aligned}
$$

where $d_{H}\left(x_{m, j}^{k} \rightarrow x_{\widehat{m}, j}^{k}\right)$ is the Hamming distance between the transmitted and the received modulated symbol, $d_{H}\left(x_{m, j}^{k} \rightarrow x_{m, \hat{j}}^{k}\right)$ is the Hamming distance between the transmitted and the received spatial symbol, and $d_{H}\left(x_{m, j}^{k} \rightarrow\right.$ $\left.x_{\widehat{m}, \hat{j}}^{k}\right)$ is the Hamming distance between the transmitted and received super symbol such that

$$
\begin{aligned}
d_{H}\left(x_{m, j}^{k} \longrightarrow x_{\widehat{m}, \hat{j}}^{k}\right)= & d_{H}\left(x_{m, j}^{k} \longrightarrow x_{\widehat{m}, j}^{k}\right) \\
& +d_{H}\left(x_{m, j}^{k} \longrightarrow x_{m, \hat{j}}^{k}\right) .
\end{aligned}
$$

The APWEP can be defined for these three cases as

$$
\begin{gathered}
\operatorname{APWEP}_{\text {symbol }}=E\left[\operatorname{Pr}\left(x_{m, j}^{k} \longrightarrow x_{\widehat{m}, j}^{k}\right)\right], \quad \widehat{m} \neq m, \\
\operatorname{APWEP}_{\text {spatial }}=E\left[\operatorname{Pr}\left(x_{m, j}^{k} \longrightarrow x_{m, \hat{j}}^{k}\right)\right], \quad \widehat{j} \neq j, \\
\mathrm{APWEP}_{\text {joint }}=E\left[\operatorname{Pr}\left(x_{m, j}^{k} \longrightarrow x_{\widehat{m}, \hat{j}}^{k}\right)\right], \quad \widehat{m} \neq m, \widehat{j} \neq j,
\end{gathered}
$$

where $E[\cdot]$ denotes the expectation operation. Next we present formulas for analyzing the APWEP for the three cases above. In this analysis we will follow the same procedure in [13], by introducing the effect of multiuser interference and channel fading in the symbol, spatial, and joint cases.

3.1. APWEP of Subchannel Selection Method. APWEP symbol may occur when the symbol is received at the intended spatial antenna $j$ but the ML detector (MLD) detects a wrong symbol

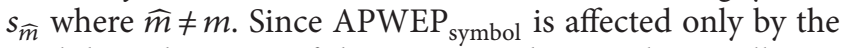
Euclidean distances of the points in the signal constellation diagram, then $\operatorname{Pr}\left(x_{m, j}^{k} \rightarrow x_{\widehat{m}, j}^{k}\right)$ may be written as [13]

$$
\operatorname{Pr}\left(x_{m, j}^{k} \longrightarrow x_{\widehat{m}, j}^{k}\right)=Q\left(\beta \frac{\left|s_{m}\right|^{2}+\left|s_{\widehat{m}}\right|^{2}-2 \operatorname{Re}\left(s_{m}^{*} s_{\widehat{m}}\right)}{\left|s_{\widehat{m}}-s_{m}\right| \sqrt{2 N_{0}}}\right)
$$

where $\operatorname{Re}(\cdot)$ and $(\cdot)^{*}$ denote the real part and the complex conjugate of a symbol, respectively. 
APWEP ${ }_{\text {spatial }}$ can occur when the MLD detects the correct symbol $s_{m}$ at a wrong receiving antenna $\hat{j}$, where $\hat{j} \neq j$. Then,

$$
\begin{aligned}
& \operatorname{Pr}\left(x_{m, j}^{k} \longrightarrow x_{m, \hat{j}}^{k}\right) \\
& =\operatorname{Pr}\left[\left\|\frac{\mathbf{y}^{k}}{\beta}-\mathbf{x}_{m, j}^{k}\right\|^{2}>\left\|\frac{\mathbf{y}^{k}}{\beta}-\mathbf{x}_{m, \hat{j}}^{k}\right\|^{2}\right] \\
& =\operatorname{Pr}\left[-\left|s_{m}\right|^{2}-\frac{2 \operatorname{Re}\left(w_{j}^{k} s_{m}^{*}\right)}{\beta}\right. \\
& \left.>\frac{2 \operatorname{Re}\left(v_{\widehat{j}}^{k} s_{m}^{*}\right)}{\beta}-\frac{2 \operatorname{Re}\left(w_{\widehat{j}}^{k} s_{m}^{*}\right)}{\beta}\right] \\
& =\operatorname{Pr}\left[\operatorname{Re}\left(w_{\widehat{j}}^{k} s_{m}^{*}\right)-\operatorname{Re}\left(w_{j}^{k} s_{m}^{*}\right)>\frac{\beta\left|s_{m}\right|^{2}}{2}-\operatorname{Re}\left(v_{\widehat{j}}^{k} s_{m}^{*}\right)\right],
\end{aligned}
$$

where $w_{j}^{k}$ and $w_{\hat{j}}^{k}$ are independent and identically distributed (i.i.d) random variables with zero mean and variance $\sigma^{2}=$ $N_{0} / 2$. Thus, $\operatorname{Re}\left(w_{\widehat{j}}^{k} s_{m}^{*}\right)-\operatorname{Re}\left(w_{j}^{k} s_{m}^{*}\right)$ is a Gaussian random variable with zero mean and variance $\sigma^{2}=\left|s_{m}\right|^{2} N_{0}$. Then $\operatorname{Pr}\left(x_{m, j}^{k} \rightarrow x_{m, \hat{j}}^{k}\right)$ can be written as

$$
\operatorname{Pr}\left(x_{m, j}^{k} \longrightarrow x_{m, \hat{j}}^{k}\right)=Q\left(\frac{\beta\left|s_{m}\right|^{2}-2 \operatorname{Re}\left(v_{\hat{j}}^{k} s_{m}^{*}\right)}{2\left|s_{m}\right| \sqrt{N_{0}}}\right)
$$

APWEP $_{\text {joint }}$ may occur when the MLD detects a wrong symbol $s_{\widehat{m}}$ at a wrong spatial antenna $\widehat{j}$, where $\widehat{m} \neq m$ and $\widehat{j} \neq j$. Then,

$$
\begin{gathered}
\operatorname{Pr}\left(x_{m, j}^{k} \longrightarrow x_{\widehat{m}, \hat{j}}^{k}\right) \\
=\operatorname{Pr}\left[\left\|\frac{\mathbf{y}^{k}}{\beta}-\mathbf{x}_{m, j}^{k}\right\|^{2}>\left\|\frac{\mathbf{y}^{k}}{\beta}-\mathbf{x}_{\widehat{m}, \hat{j}}^{k}\right\|^{2}\right] \\
=\operatorname{Pr}\left[-\left|s_{m}\right|^{2}-\frac{2 \operatorname{Re}\left(w_{j}^{k} s_{m}^{*}\right)}{\beta}\right. \\
\left.>\left|s_{\widehat{m}}\right|^{2}-\frac{2 \operatorname{Re}\left(v_{\widehat{j}}^{k} s_{m}^{*}\right)}{\beta}-\frac{2 \operatorname{Re}\left(w_{\widehat{j}}^{k} s_{m}^{*}\right)}{\beta}\right] \\
=\operatorname{Pr}\left[\operatorname{Re}\left(w_{\widehat{j}}^{k} s_{\widehat{m}}^{*}\right)-\operatorname{Re}\left(w_{j}^{k} s_{m}^{*}\right)\right. \\
\left.>\beta \frac{\left|s_{m}\right|^{2}+\left|s_{\widehat{m}}\right|^{2}}{2}-\operatorname{Re}\left(v_{\widehat{j}}^{k} s_{\widehat{m}}^{*}\right)\right],
\end{gathered}
$$

where $\operatorname{Re}\left(w_{\widehat{j}}^{k} s_{\widehat{m}}^{*}\right)-\operatorname{Re}\left(w_{j}^{k} s_{m}^{*}\right)$ is a Gaussian random variable with zero mean and variance $\sigma^{2}=\left(\left|s_{m}\right|^{2}+\left|s_{\widehat{m}}\right|^{2}\right) N_{0} / 2$. The final expression for this case is given by

$$
\begin{aligned}
& \operatorname{Pr}\left(x_{m, j}^{k} \longrightarrow x_{\widehat{m}, \hat{j}}^{k}\right) \\
& =Q\left(\frac{\beta\left(\left|s_{m}\right|^{2}+\left|s_{\widehat{m}}\right|^{2}\right)-2 \operatorname{Re}\left(v_{\widehat{j}}^{k} s_{\widehat{m}}^{*}\right)}{\sqrt{\left|s_{m}\right|^{2}+\left|s_{\widehat{m}}\right|^{2}} \sqrt{2 N_{0}}}\right) .
\end{aligned}
$$

In (23) and (25), the terms which contain $v_{\hat{j}}^{k}$ are the contributions of multiuser interference and channel fading, as a result of the new considerations in this paper. The subtraction of this term from the argument of the Q-function implies that the overall APWEP will be higher or degraded (since $Q(x)>Q(y)$ if $x<y)$ compared to MU-MIMO without SM.

3.2. APWEP of Zero-Padding Method. In this method, the effect of multiuser interference and channel fading is totally cancelled. Using similar steps above to obtain the APWEP formulas for the zero-padding method, we found that $\operatorname{Pr}\left(x_{m, j}^{k} \rightarrow x_{\widehat{m}, j}^{k}\right)$ is exactly expressed as in (22), while $\operatorname{Pr}\left(x_{m, j}^{k} \rightarrow x_{m, \hat{j}}^{k}\right)$ and $\operatorname{Pr}\left(x_{m, j}^{k} \rightarrow x_{\widehat{m}, \hat{j}}^{k}\right)$ may be, respectively, expressed as in (24) and (26) by eliminating the contribution of the multiuser interference and channel fading. Thus we can write for this case that

$$
\begin{gathered}
\operatorname{Pr}\left(x_{m, j}^{k} \longrightarrow x_{m, \hat{j}}^{k}\right)=Q\left(\beta \frac{\left|s_{m}\right|}{\sqrt{N_{0}}}\right), \\
\operatorname{Pr}\left(x_{m, j}^{k} \longrightarrow x_{\widehat{m}, \hat{j}}^{k}\right)=Q\left(\frac{\beta \sqrt{\left|s_{m}\right|^{2}+\left|s_{\widehat{m}}\right|^{2}}}{\sqrt{2 N_{0}}}\right) .
\end{gathered}
$$

Then, the error probability given by (26) and (27) is less than that given by (23) and (25) which means that the zeropadding method can improve the total ABER performance.

Throughput and Complexity Analysis. We will quantify the system throughput in terms of the effective number of transmitted bits per channel use (bpcu), while the complexity is characterized by the total number of multiplications required at the MLD. Because we considered here the case where only one antenna is activated per user, we will compare these parameters with that of the conventional multiuser MIMO system with one data stream per user $\left(N_{r}=1\right)$. The effective number of bits per channel use for MU-SM is given by

$$
n_{\mathrm{MU}-\mathrm{SM}}=K\left[\log _{2}(M)+\log _{2}\left(N_{r}\right)\right]
$$

while the effective number of bits per channel use for conventional MU-MIMO is $n_{\mathrm{MU}-\mathrm{MIMO}}=K \log _{2}(M)$. Then, 
TABLE 1: The increase in throughput and complexity of MU-SM relative to those of conventional MU-MIMO.

\begin{tabular}{lccc}
\hline$M$ & $N_{r}$ & $\begin{array}{c}\text { Relative increase in throughput } \\
\left(\left(\log _{2}\left(N_{r}\right) / \log _{2}(M)\right) \times 100 \%\right)\end{array}$ & $\begin{array}{c}\text { Relative increase in complexity } \\
\left(\left(N_{r} / 2 M\right) \times 100 \%\right)\end{array}$ \\
\hline 2 & 2 & 100 & 50 \\
2 & 4 & 200 & 25 \\
\hline 4 & 2 & 50 & 50 \\
4 & 4 & 100 & 100 \\
4 & 8 & 150 & 12.5 \\
\hline 8 & 2 & 33.33 & 25 \\
8 & 4 & 66.67 & 50 \\
8 & 8 & 100 & 100 \\
8 & 16 & 133.33 & 6.25 \\
16 & 2 & 25 & 12.5 \\
16 & 4 & 50 & 25 \\
16 & 8 & 75 & 50 \\
\hline
\end{tabular}

the throughput of MU-SM relative to that of the conventional MU-MIMO is given by

$$
\begin{aligned}
R_{\mathrm{rel}} & =\frac{n_{\mathrm{MU}-\mathrm{SM}}}{n_{\mathrm{MU}-\mathrm{MIMO}}} \\
& =1+\frac{\log _{2}\left(N_{r}\right)}{\log _{2}(M)},
\end{aligned}
$$

where $\log _{2}\left(N_{r}\right) / \log _{2}(M)$ is the relative increase in the throughput. As shown in [13], separate detection can be used in SM system in which the spatial symbol $j$ and the modulated symbol $s_{m}$ are detected separately such that

$$
\widehat{j}=\arg \max _{j \in\left\{1,2, . ., N_{r}\right\}}\left\{\left|y_{j}^{k}\right|^{2}\right\},
$$

where $y_{j}^{k}$ is the $j$ th element of $\mathbf{y}^{k}$.

$$
\begin{aligned}
\widehat{m} & =\arg \min _{m \in\{1,2, . ., M\}}\left\{\left|\frac{1}{\beta} y_{\hat{j}}^{k}-s_{m}\right|^{2}\right\} \\
& =\arg \min _{m \in\{1,2, . ., M\}}\left\{2 \operatorname{Re}\left(\frac{1}{\beta} y_{\widehat{j}}^{k} s_{m}\right)-\left|s_{m}\right|^{2}\right\} .
\end{aligned}
$$

As explained above, the correct decision is obtained when $\widehat{m}=m$ and $\widehat{j}=j$. The number of multiplications required in (30) to get $\hat{j}$ is $N_{r}$ while the number of multiplications required in (31) to get $s_{\widehat{m}}$ is $2 M$. The MLD computational complexity for MU-SM, which is defined here as the total number of multiplications required for the detection process, can be expressed as

$$
C_{\mathrm{MU}-\mathrm{SM}}=N_{r}+2 M
$$

whereas the MLD computational complexity of the conventional MU-MIMO system with one receiving antenna for each user is $C_{\mathrm{MU}-\mathrm{MIMO}}=2 \mathrm{M}$, because there is no spatial symbol to be detected in this case. Thus, the relative MLD complexity for the MU-SM versus the conventional MUMIMO is

$$
\begin{aligned}
C_{\text {rel }} & =\frac{C_{\text {MU-SM }}}{C_{\text {MU-MIMO }}} \\
& =1+\frac{N_{r}}{2 M},
\end{aligned}
$$

where $N_{r} / 2 M$ is the relative increase in complexity. Table 1 presents a tabulation of the relative increase in throughput and complexity for MU-SM in comparison with the conventional MU-MIMO system. From this table, it is observed that significant throughput enhancement can be achieved in the proposed scheme at moderate complexity. Also noticeable from this table is the lack of dependence of the throughput and complexity increase on the BSTx antennas, $N_{t}$. This is because we are comparing with another MU-MIMO system that has the same number of BSTx antennas.

\section{Simulation Results}

In this section, we present simulation results for the bit error rate (BER) performance of the proposed SM for multiuser MIMO scheme. The BER curves presented in all figures are averaged over all users. In the simulations, we considered QPSK and 16-QAM modulation formats. Figure 3 compares the analytical results of (17)-(25) with simulation, where it is easily observed that both analytical and simulation results have very close matching (especially in high SNR). This figure also depicts the effect of increasing the number of receiving antennas for each user on the average (BER) performance. In Figure 3, we considered transmitting antennas for cases of 2 and 4 users, with different number of receiving antennas for each user. As shown in the figure, the BER performance of the MU-SM system degrades as the number of receiving antennas $N_{r}$ for each user increases. This is due to the fact that 


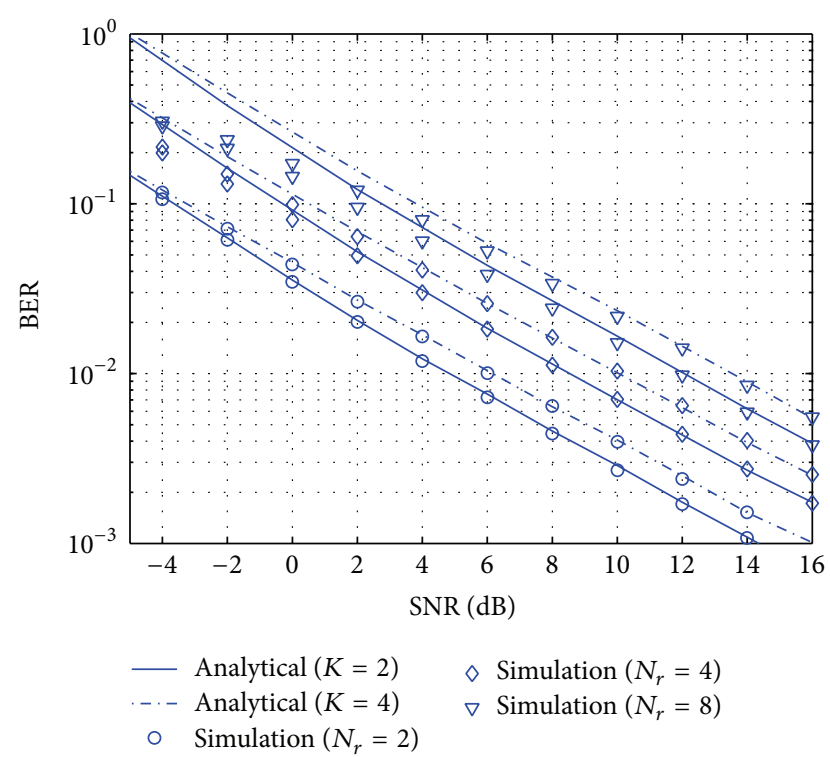

FIGURE 3: BER performance of subchannel selection method with different number of receiving antennas for $N_{t}=10, K=2$, and $K=4$ for QPSK modulation.

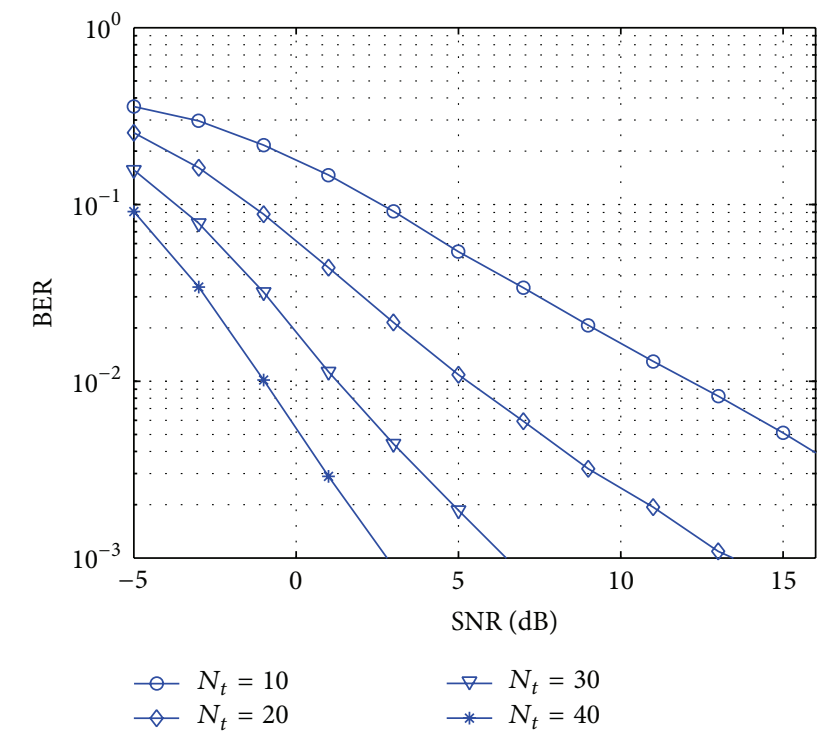

FIGURE 4: BER Performance of subchannel selection method with different number of transmit antennas for $K=5$ and $N_{r}=2$ for QPSK modulation.

the multiple receiving antennas are not used for diversity but for SM. The receiver would not know ahead what antenna is intended to be switched by the BSTx. Thus, the ML detection is applied over all the receiving antennas, which also increase the probability of error in the received data with increasing $N_{r}$ for each user.

Figure 4 shows the BER performance of a multiuser MIMO system with spatial modulation at the receiver side for different number of transmitting antennas. In this figure, we considered 5 users, with $N_{r}=2$ per user, and $N_{t}=$

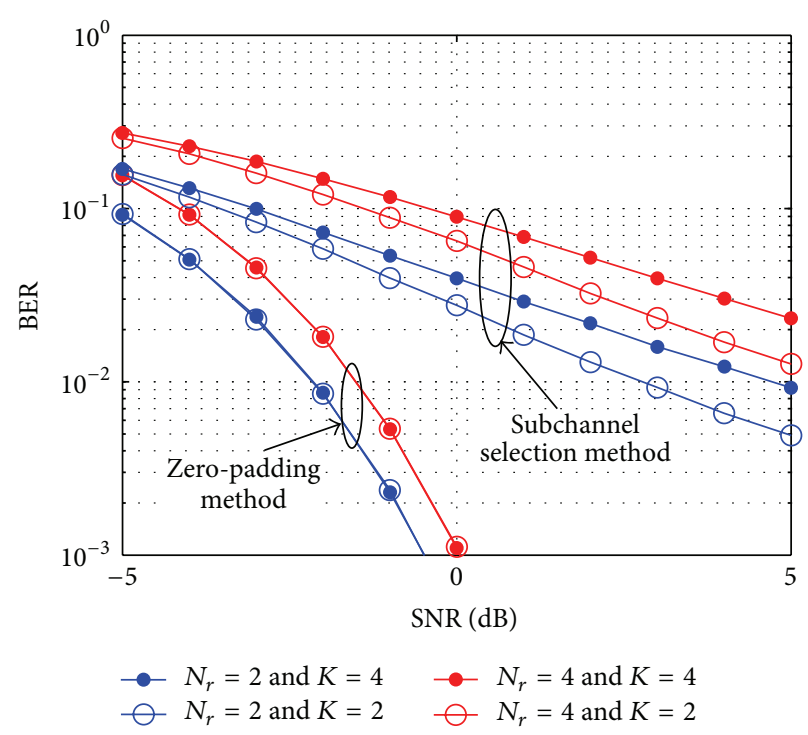

FIGURE 5: BER of subchannel selection method and zero-padding method with $K=2$ and $K=4$ and $N_{t}=16$ for QPSK modulation.

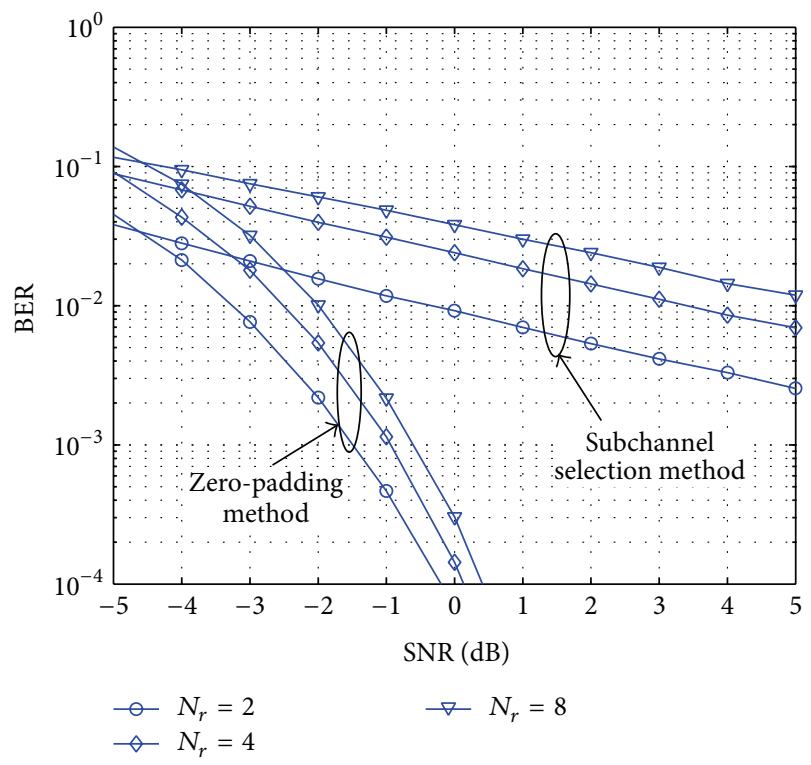

FIGURE 6: BER of subchannel selection method and zero-padding method with massive BSTx antennas $\left(N_{t}=50\right), K=5$, for 16-QAM modulation.

$10,20,30$, and 40 . As shown in this figure, the BER performance gets better when the number of transmitting antennas increases, due to the diversity gain achieved at the transmitter. Figure 5 compares the BER performance for the two proposed methods. As shown in the figure, zero-padding method gives a significant improvement in the BER performance over subchannel selection method. The figure also shows that in the zero-padding method the BER performance is not affected by the number of users because there is no multiuser interference in this case. Figure 6 shows the BER performance results of the two proposed methods for 16-QAM modulation 
with a large number of transmitting antennas at the BSTx. It is observed from this figure that despite using higher constellation (16-QAM) and higher number of users $(K=5)$ compared to the results in Figure 5, the BER performance is not too much degraded due to the extra diversity effects provided by the massive number of BSTx antennas used in Figure 6.

\section{Conclusion}

In this paper we present the concept of spatial modulation (SM) scheme for massive multiuser MIMO (MU-MIMO) system. In this scheme, the index of the active receiving antenna of each user in a MU-MIMO system is used to convey extra information in addition to the transmitted symbols. Simulation results show that significant increase in the system throughput is achieved as the number of available receiving antennas per user is increased. Two methods are proposed for implementing the SM scheme for massive MU-MIMO system: subchannel selection method and zero-padding method. BER performance of the proposed methods are also studied. Our results show that for the subchannel selection method, the BER performance degrades with increasing the number of users serviced by the BSTx or the number of receiving antennas per user. For the zeropadding method, increasing the number of users does not affect the BER performance since the multiuser interference is totally removed by the combination of zero-padding and precoding operations applied at the BSTx.

\section{Conflict of Interests}

The authors declare that there is no conflict of interests regarding the publication of this paper.

\section{Acknowledgment}

This work was supported by NSTIP strategic technologies programs (number 11-ELE1854-02) in the Kingdom of Saudi Arabia.

\section{References}

[1] J. Mietzner, R. Schober, L. Lampe, W. Gerstacker, and P. Hoeher, "Multiple-antenna techniques for wireless communicationsa comprehensive literature survey," IEEE Communications Surveys and Tutorials, vol. 11, no. 2, pp. 87-105, 2009.

[2] F. Rusek, D. Persson, B. Lau, and E. Larsson, "Scaling Up MIMO: opportunities and challenges with very large arrays," IEEE Signal Processing Magazine, vol. 30, no. 1, pp. 40-60, 2013.

[3] A. Akhlaq, A. I. Sulyman, H. Hassanein, A. Alsanie, and S. Alshebeili, "Performance analysis of relay multiplexing scheme in cellular systems employing massive MIMOantennas," IET Communications. In press.

[4] J. Hoydis, S. Ten Brink, and M. Debbah, "Massive MIMO in the UL/DL of cellular networks: how many antennas do we need?" IEEE Journal on Selected Areas in Communications, vol. 31, no. 2, pp. 160-171, 2013.
[5] M. Di Renzo, H. Haas, and P. M. Grant, "Spatial modulation for multiple-antenna wireless systems: a survey," IEEE Communications Magazine, vol. 49, no. 12, pp. 182-191, 2011.

[6] Y. Zhang, C. Ji, W. Q. Malik, D. C. O’Brien, and D. J. Edwards, "Receive antenna selection for MIMO systems over correlated fading channels," IEEE Transactions on Wireless Communications, vol. 8, no. 9, 2009.

[7] R. Rajashekar, K. V. S. Hari, and L. Hanzo, "Antenna selection in spatial modulation systems," IEEE Communications Letters, vol. 17, no. 3, pp. 521-524, 2013.

[8] Y. Yang and B. Jiao, "Information-guided channel-hopping for high data rate wireless communication," IEEE Communications Letters, vol. 12, no. 4, pp. 225-227, 2008.

[9] R. Y. Mesleh, H. Haas, S. Sinanović, C. W. Ahn, and S. Yun, "Spatial modulation," IEEE Transactions on Vehicular Technology, vol. 57, no. 4, pp. 2228-2241, 2008.

[10] J. Jeganathan, A. Ghrayeb, L. Szczecinski, and A. Ceron, "Space shift keying modulation for MIMO channels," IEEE Transactions on Wireless Communications, vol. 8, no. 7, pp. 3692-3703, 2009.

[11] E. Basar, U. Aygolu, E. Panayici, and H. V. Poor, "Spacetime block coded spatial modulation," IEEE Transactions on Communications, vol. 59, pp. 823-832, 2010.

[12] J. Wang, S. Jia, and J. Song, "Generalised spatial modulation system with multiple active transmit antennas and low complexity detection scheme," IEEE Transactions on Wireless Communications, vol. 11, no. 4, pp. 1605-1615, 2012.

[13] R. Zhang, L. Yang, and L. Hanzo, "Generalised pre-coding aided spatial modulation," IEEE Transactions on Wireless Communications, vol. 12, no. 11, pp. 5434-5443, 2013.

[14] M. Di Renzo and H. Haas, "Bit error probability of SMMIMO over generalized fading channels," IEEE Transactions on Vehicular Technology, vol. 61, no. 3, pp. 1124-1144, 2012. 

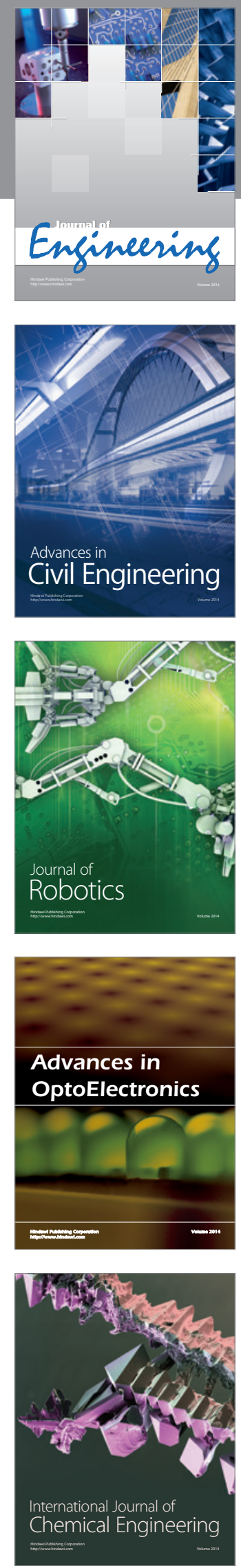

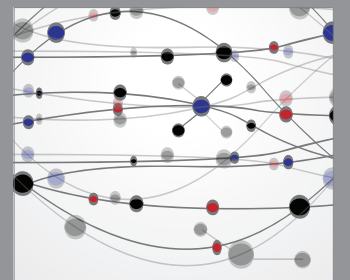

The Scientific World Journal
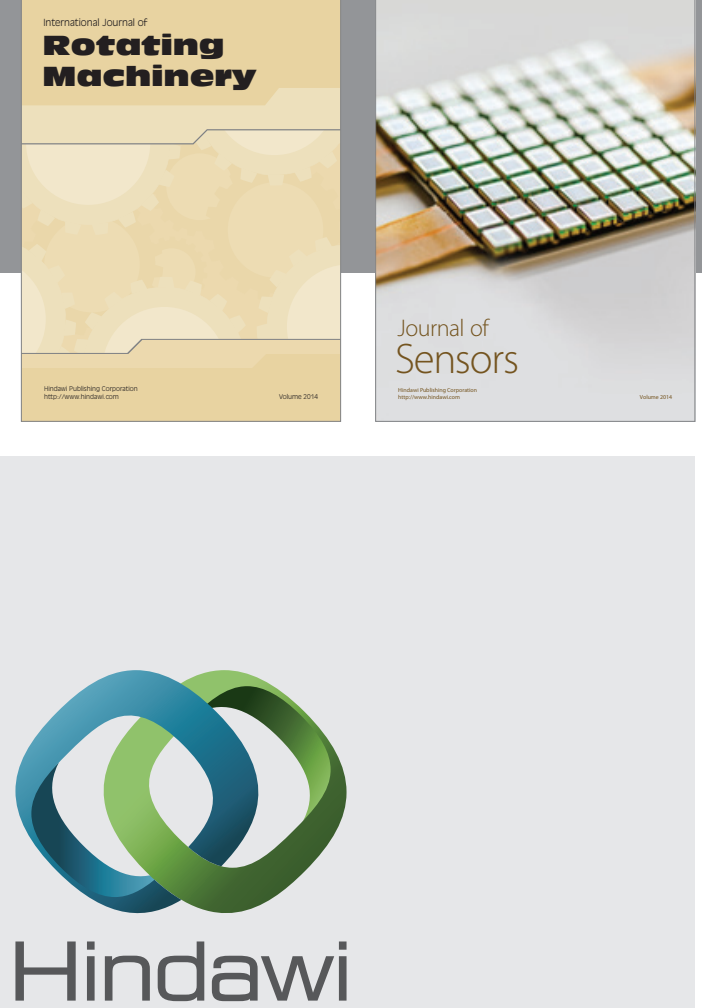

Submit your manuscripts at http://www.hindawi.com
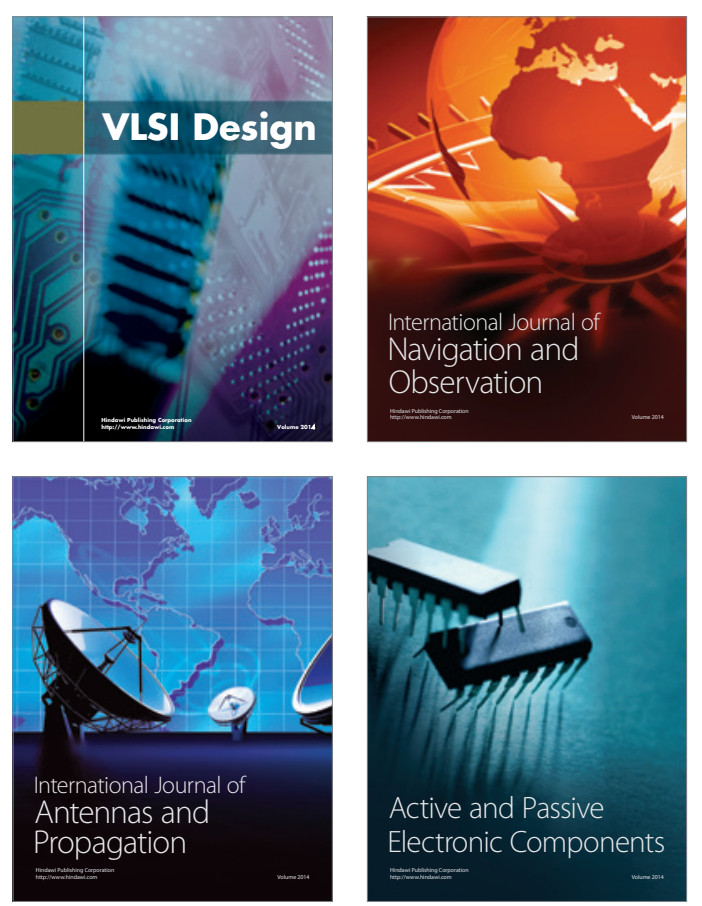
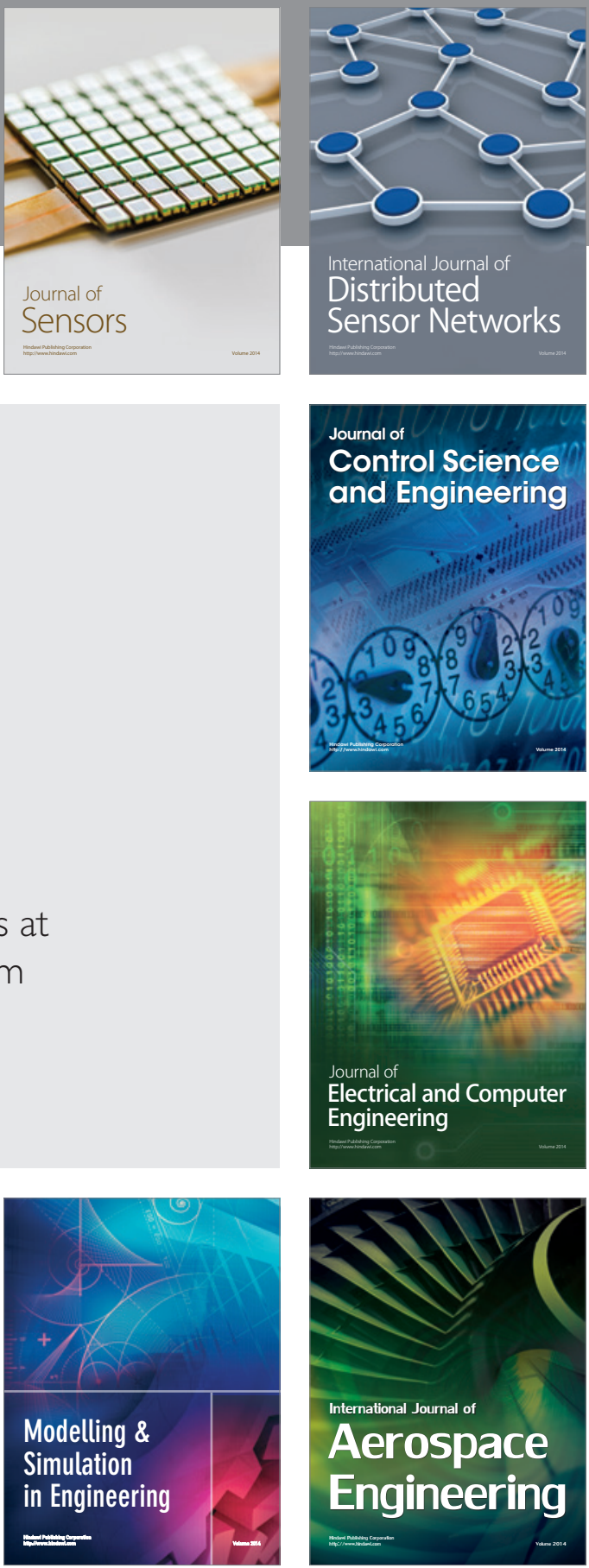

Journal of

Control Science

and Engineering
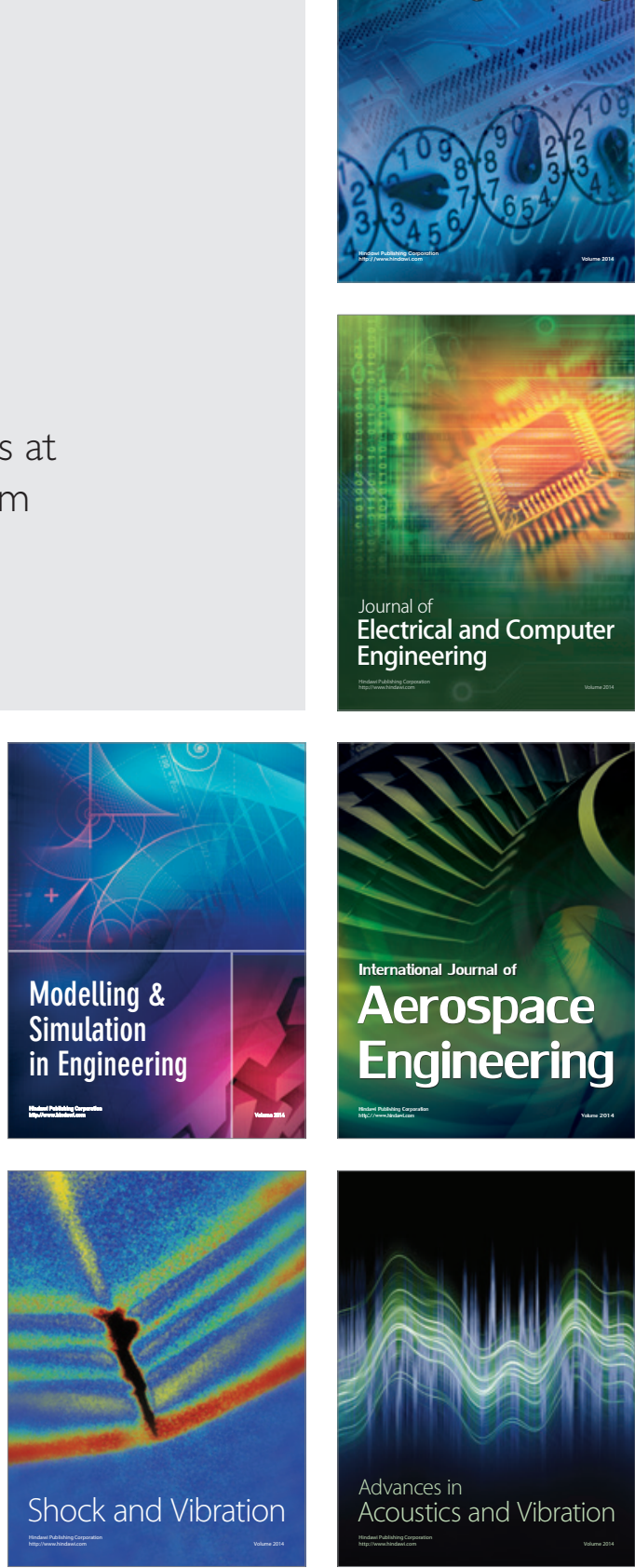